\title{
Kernos
}

Revue internationale et pluridisciplinaire de religion grecque antique

6 | 1993

Varia

\section{Lucrèce et les demeures des dieux}

Un symbolisme à portée épicurienne

\section{Pierre-Jacques Dehon}

\section{(2) OpenEdition \\ 1 Journals}

\section{Édition électronique}

URL : http://journals.openedition.org/kernos/536

DOI : $10.4000 /$ kernos.536

ISSN : 2034-7871

Éditeur

Centre international d'étude de la religion grecque antique

\section{Édition imprimée}

Date de publication : 1 janvier 1993

Pagination : 67-77

ISSN : 0776-3824

\section{Référence électronique}

Pierre-Jacques Dehon, « Lucrèce et les demeures des dieux », Kernos [En ligne], 6 | 1993, mis en ligne le 07 avril 2011, consulté le 20 avril 2019. URL : http://journals.openedition.org/kernos/536 ; DOI :

10.4000/kernos.536 
Kernos, 6 (1993), p. 67-77.

\section{LUCRÈCE ET LES DEMEURES DES DIEUX :}

\section{UN SYMBOLISME À PORTÉE ÉPICURIENNE}

L'éloge d'Épicure qui constitue le prologue au livre III du De rerum natura lucrétien (v. 1-30) est une invocation composée de deux parties : la première (v. 1-13) est un vibrant hommage au Maître; la seconde (v. 14-30) exalte son enseignement et rappelle l'essence de sa doctrine en matière de religion. Les vers 18 à 24 , qui retiendront plus spécialement notre attention ${ }^{1}$, dépeignent les demeures des dieux (sedes, v. 18), sises, selon les épicuriens, dans les espaces entre les différents mondes

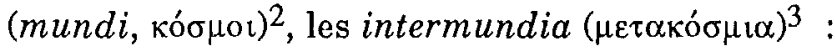

Apparet diuum numen sedesque quietae,

Quas neque concutiunt uenti, nec nubila nimbis

Aspergunt, neque nix acri concreta pruina

Cana cadens uiolat, semperque innubilus aether

Integit, et large diffuso lumine rident.

Omnia suppeditat porro natura neque ulla

Res animi pacem delibat tempore in ullo.

Je voudrais consacrer les pages suivantes à montrer, dans un premier temps, que cette description, inspirée d'un portrait de l'Olympe

1 Sur ce passage, voir H.A.J. MuNRo, T. Lucreti Cari De rerum natura libri sex, ed. with n. and a transl., vol. II, 4th rev. ed., New York-London, 1978 (= 1886), p. 178; R. Heinze, T. Lucretius Carus. De rerum natura Buch III, erklärt, Leipzig, 1897, p. 52-53; A. ERNouT-L. RoBIN, Lucrèce. De rerum natura. Commentaire exégétique et critique. Tome deuxième. Livres III et IV, Paris, 1926, p. 5-6; C. B AILEY, Titi Lucreti Cari De rerum natura libri sex, ed. with prolegomena, crit. app., transl. and comm., vol. II, Oxford, 1950, p. 990-991; E.J. K ENNEY, Lucretius. De rerum natura. Book III, ed., Cambridge, 1971, p. 77-79. Sur la philosophie de Lucrèce, voir en particulier P. BOYANCÉ, Lucrèce et l'épicurisme, Paris, 1963.

2 Cf. LUCR , V, 528 : in uariis mundis uaria ratione creatis.

3 Voir A. ERNOUT-L. RoBIN, op. cit., p. 5; J. BRUN, L'épicurisme, Paris, 1959, p. 87; E.J. KeNNEY, op. cit., p. 77-78. Lucrèce écrit ailleurs : Illud item non est ut possis credere, sedes / Esse deum sanctas in mundi partibus ullis (V, 146-147). 
figurant dans l'Odyssée (VI, 42-46) ${ }^{4}$, est orientée par une idée directrice qui la sous-tend : celle de l'hiver et, plus précisément, celle de l'opposition entre hiver et printemps; ensuite, je m'efforcerai d'expliquer comment une utilisation symbolique de l'hiver permet à Lucrèce de fonder les grands principes épicuriens relatifs à la divinité.

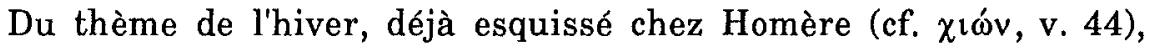
Lucrèce a fait la notion de base d'une argumentation implicite : nous verrons qu'il suggère d'abord cette idée de façon discrète, puis, par une gradation, la rend de plus en plus sensible. Avant tout, le poète invite le lecteur à partager une apparition (apparet, v. 18; cf. uideo, v. 17) ${ }^{5}$, voire une initiation. Le caractère solennel de la révélation est souligné par l'abondance de spondées (v. 18) et la notion importante est solidement encadrée par les césures (diuum numen, v. 18). Car ce sont des informations sur le diuum numen qu'en définitive le portrait des sedes... quietae apportera au lecteur. Lucrèce se livre à une véritable mise en scène : les remparts du monde s'écartent, tels les rideaux d'un théâtre (mœnia mundi / discedunt, v. 16-17).

La caractéristique première du domaine des dieux - et de ses occupants - est soulignée par quietae (v. 18) : calme, tranquillité, apaisement, éventuellement sur le plan spirituel (cf. animi pacem, v. 24). Mais quietus, qualificatif dont Lucrèce use par deux fois à propos des dieux (V, 168 et VI, 73), se dit notamment de la mer ou d'une atmosphère libre de tempêtes ${ }^{6}$. Or le thème de la tempête est lié à celui de

4 On s'accorde souvent à y déceler une interpolation : voir à ce sujet V. BÉRARD, Introduction à l'Odyssée. Tome II. Le poème représenté. Le poème édité, $2 \mathrm{e}$ éd. rev. et corr., Paris, 1933, p. 53-57, et l'apparat critique de son édition, L'Odyssée. «Poésie homérique». Tome I : chants I-VII, texte ét. et trad., 5e éd., Paris, 1955, p. 169. Cf. cependant W.B. STANFORD, The Odyssey of Homer, ed. with general and grammatical intr., comm., and ind., vol. I, 2nd ed., London-MelbourneToronto-New York, 1967, p. 310. Sur les mérites respectifs des deux passages, voir E. BIGNONE, Storia della letteratura latina. Volume secondo. La prosa romana fino all'età di Cesare. Lucilio, Lucrezio, Catullo, Firenze, s.d. [1946], p. 254-256; D.E.W. WormeLl, The Personal World of Lucretius, in Lucretius, ed. by D.R. DUDLEY, London, 1967 (= 1965), p. 35-67, sp. 45; D. WEST, The Imagery and Poetry of Lucretius, Edinburgh, 1969, p. 31-33; Fr. GIANCOTTI, Il preludio di Lucrezio e altri scritti lucreziani ed epicurei, Messina-Firenze, s.d. [1978], p. 87-90.

5 Voir A. ERNOUT-L. RoBin, op. cit., p. 5-6.

6 Cf. CÉs., B. C., III, 6, 3 (quietam... stationem); VIRG., Én., V, 848 (fluctus... quietos); Hor., Epo., X, 11 (quietione... aequore); Ov., Mét., V, 6 (freto..., quod... quietum). 
l'hiver : l'emploi du même terme, hiems, pour désigner tantôt l'une, tantôt l'autre en témoigne ${ }^{7}$. Le Mantouan empruntera l'expression lucrétienne sedes... quietae ${ }^{8}$ et l'appliquera au Latium, région de cette Italie où, écrit-il ailleurs ( $G$., II, 149), règne un éternel printemps (uer adsiduom), endroit "divin" par excellence, puisque choisi par les destins (Én., I, 205-206 : Latium sedes ubi fata quietas / ostendunt).

L'idée de l'absence d'intempéries se précise par la suite, dans des vers (19-21) où l'accumulation de négations contribue, comme chez

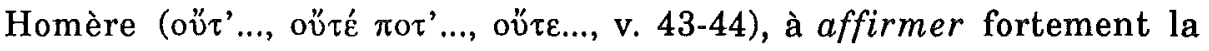
sérénité des sedes (neque..., nec..., neque...). Lucrèce observe en

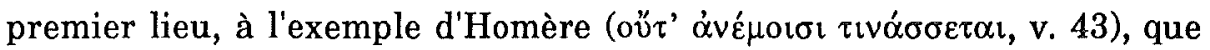
les vents ne s'y font pas sentir (quas neque concutiunt uenti, v. 19). Ces derniers soufflent en toute saison, mais leur action déterminante en hiver a marqué les poètes latins : on rencontre uenti en contexte hivernal chez un Ovide ${ }^{9}$; du reste, l'influence de certains vents, l'Aquilon ou Borée ${ }^{10}$, le Caurus ${ }^{11}$, l'Auster ou Notus ${ }^{12}$, le Vulturne ou Eurus ${ }^{13}$, est habituellement notée dans les évocations de la saison. Quant au verbe qu'utilise Lucrèce pour définir le rôle des vents $(\text { concutio })^{14}$, il apparait dans un passage de son œuvre traitant du froid

7 Cf. e.g., pour hiems-«hiver», ENN., Ann., 420 (S KUTSCH); LUCR., V, 747; VIRG., G. , I, 299; pour hiems-«tempête", ACC., Médée, 413 (RIBBECK); VIRG., En., IV, 52; Ov., Hér., VII, 41; MANIL., II, 104. Voir à ce sujet A. WALDE-J.B. HoFmanN, Lateinisches etymologisches Wörterbuch. Erster Band (A-L), Heidelberg, 1938, p. 645, et A. ERNOUT-A. MEILLET, Dictionnaire étymologique de la langue latine (Histoire des mots), 4e éd. rev., corr. et augm. d'un index, Paris, 1959, p. 293.

8 Cf. aussi Albinovanus Pedo, Fr., 21-23 (Buechner) : Aliena quid aequora remis / Et sacras uiolamus aquas diuumque quietas / Turbamus sedes?

9 Mét., I, 120 (uentis glacies adstricta pependit, au sein d'un tableau peignant la fin de l'âge d'or [fait non dénué d'intérêt : cf. infra, p. 75-76]) et Tr., III, 10, 29 . 30 (Caeruleos uentis latices durantibus Hister / congelat).

10 Cf. e.g. VIRG., En., III, 285; HoR., Epo., XIII, 3; OV., Ib. , 201; Tr., III, 10, 11; Colum., X, 76; Luc., IV, 50; Sil., XII, 7; STACE, Silv., IV, 5, 8; MART., I, 49, 20.

11 Cf. e.g. Virg., G., III, 356; Colum., X, 75; S ÉN., Méd., 412; SiL., III, 523.

12 Cf. e.g. Cic., Ph., XXXIII, 67 (Soubiran); Tib., I, 1, 47; Germ., Ph., 293; PerSe, VI, 12; SIL., XII, 2; JUV., IV, 59.

13 Cf. VIRG., G., II, 339; III, 382; ColuM., X, 76; JUV., XIV, 186; mais voir ma note Horace, Odes, I, 25, 19-20, in Latomus, 50 (1991), p. 184-186, sp. 185.

14 En VI, 592-593, il écrit : uis uenti... /... incutit inde tremorem. 
(VI, 594-595 : Frigus uti nostros penitus cum uenit in artus, / Coneutit inuitos) ${ }^{15}$.

Après les vents, Lucrèce cite les pluies, comme dans le modèle

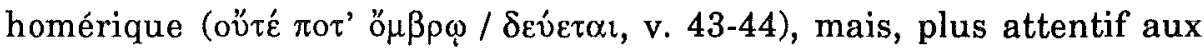
phénomènes physiques et météorologiques ${ }^{16}$, il ajoute une mention des nuages qui produit une allitération (nec nubila nimbis / aspergunt, v. 19-20). Certes, il peut s'agir de n'importe quelles pluies, mais il n'est pas rare de trouver, dans les lettres latines, des allusions aux pluies hivernales ${ }^{17}$. Petit à petit semble se dessiner en filigrane l'image de l'hiver, ou plus exactement de son opposé.

Le point suivant concerne l'absence de précipitations neigeuses; le

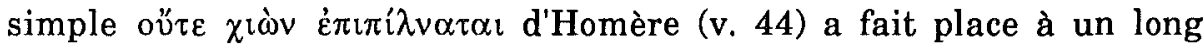
développement : neque nix acri concreta pruina / Cana cadens uiolat (v. 20-21). La fin du vers 20 contient, précédés d'une négation, quatre mots relevant du lexique hivernal : le substantif nix est employé dans les portraits de la saison pour désigner la neige ${ }^{18}$; acris (acer) figure parmi les épithètes que les poètes ont accordées à hiems ${ }^{19}$; le participe parfait passif concretus est un terme technique utilisé pour qualifier une chose durcie par le gel ou transformée en glace ${ }^{20}$ - c'est notre "gelé» -; pruina, proprement "gelée blanche» (liée à la rosée), se rencontre dans

15 Cf. aussi CIC., Ph., XXXIII, 68 (S. : Tum fixum tremulo quatietur frigore corpus, dans un tableau de l'hiver en mer) et Ov., Mét., VI, 691 (Vi freta concutio nodosaque robora uerto, dans une réplique de Borée, vent typiquement hivernal).

16 Voir D. West, op. cit. , p. 32-33, et E.J. KenNEY, op. cit. , p. 78.

17 En poésie, cf. Ov., Mét., IX, 105-106 (nimbis hiemalibus) et les évocations des imbres de l'hiver dans Hor., Epo., II, 29-30; MaNIL., II, 195; Priap., 63, 4 (BÜCHELER-HERAEUS); cf. aussi l'épithète de nature aquosa attribuée par Virgile à hiems (B., X, 66). En prose, cf. les mentions des pluies, e.g., dans SALL., Jug., 37, 4; Colum., II, 4, 4; XI, 3, 10; XII, 52, 17; SÉn., Ad Luc., 90, 16; Pline, $H$. N. , XVII, 17; XXXI, 50; FronTIN, Aq., 90, 2; TAC., Hist. , III, 69, 10.

18 Cf. e.g. LuCR., V, 746; Fur. BibaC., Fr., 15 (Buechner); VIRG., G., I, 310; Hor., Epo. , II, 30; OV., Tr. , III, 10, 11; MANL., II, 419.

19 ENN., Ann., 420 (S.); LUCR ., VI, 373; HoR., O., I, 4, 1.

20 Cf. VIRG., G., II, 318; LIV., XXI, 36, 8; Ov., Tr., III, 10, 31-32; CURT., VIII, 4, 6; SIl., III, 518; MART., IV, 3, 4; G ELL., XIX, 7, 15. Pour d'autres formes de concresco au sens de «se geler", cf. e.g. Lucr., VI, 845-846; VIRG., G., III, 360; OV., Mét. , IX, 220-221; Pont. , IV, 7, 7; Tr., III, 10, 25; 12, 29. Le verbe est généralement accompagné d'un substantif à l'ablatif instrumental indiquant la cause du durcissement : soit frigore, soit glacie, éventuellement pruinā. 
certains textes traitant de l'hiver, avec des sens variés et souvent difficiles à définir ("frimas, neige, givre, etc.») ${ }^{21}$. La référence à l'hiver devient plus nette; elle se confirme par la suite : cana, qui détermine $n i x$, est l'épithète traditionnelle de la neige ${ }^{22}$. L'adjectif peut aussi qualifier d'autres phénomènes hivernaux ${ }^{23}$, voire la saison ellemême ${ }^{24}$. Soulignons d'ores et déjà - nous verrons plus loin en quoi cela importe - que canus se dit de la tête ou des cheveux d'un vieillard ${ }^{25}$ et qu'Ovide personnifie l'hiver sous les traits d'un vieillard chenu ${ }^{26}$. Relevons également au passage le verbe uiolat, très fort, qui n'a pas d'équivalent dans le morceau homérique et est chargé d'une profonde connotation religieuse : il s'emploie à propos d'une personne qui dérange la sainteté d'un lieu sacré, qui profane ce lieu ${ }^{27}$; Lucrèce en use dans le cas d'une profanation de la divinité ${ }^{28}$. Violent contraste donc entre la pureté de la neige, soulignée par cana, et l'impureté de son action. Le verbe occupe une position importante : la césure qui le suit est le pivot du texte; elle marque une transition entre une série d'éléments

21 Cf. CIC., N. D., II, 26 (niue pruinaque concresceret); VIRG., G. , II, 376 (frigora... cana concreta pruina); PÊTR., S., CXXII, 150 (glacie concreta rigent hiemisque pruinis).

22 Cf. e.g. Fur. B IBAC., Fr, 15 (B.); HoR., S., II, 5, 41; SÉN., Épigr. , 68, 6 (PRATo = A. L. , 461, 6 [RIESE]); Phèdre, 935; Thy., 118; voir A. ERNOUT-L. RoBIN, op. cit. , p. 6, et J. ANDRÉ, Etude sur les termes de couleur dans la langue latine, Paris, 1949 , p. 67 (cf. p. 64-69).

23 Cf. e.g. VIRG., G., II, 376 (cana.. pruina); III, 442-443 (horrida cano / bruma gelu); Hor., O., I, 4, 4 (canis... pruinis); Ov., Hér., V, 16 (cana pruina); ColuM., X, 74 (canis... pruinis); SÉN., H. F., 139 (cana pruina); SIL., III, 479 (cana... grandine).

24 Cf. cana... bruma dans Ś́N., Epigr. , 3, 4 (PR. = A. L., 237, 4 [R.]); Phèdre, 966; Stace, Théb. , IV, 833-834; Avién., Arat., 613.

25 Cf. e.g. PL., Bacch., 1208 (canis capitibus); Cas., 518 (cano capite); CATUL., 64, 350 (canos... crines); HoR., O. , II, 11, 15 (canos... capillos); TIB., I, 1, 72 (cano capite); OV., Mét., IV, 474 (canos... capillos); XIII, 427 (canum... crinem); MART., IV, 53, 3-4 (cana... /.. coma).

26 Mét. , II, 30 (Et glacialis Hiems, canos hirsuta capillos); cf. aussi son portrait du Notus, vent hivernal (Mét., I, 266 : Barba grauis nimbis, canis fluit unda capillis).

27 Cf. e.g. Cı́., Rabir., 7; Vatin., 32; Ov., Ib., 617; Mét., VIII, 741; Ciris, 141 (sedem).

28 II, 614-615 (numen qui uiolarint / matris) et VI, 71-72 (non quo uiolari summa deum uis / possit); cf. aussi CIC., Dom., 140 (diuinum numen). 
négatifs et une nouvelle série d'éléments, affirmatifs cette fois ${ }^{29}$. On observera que cette transition s'opère en douceur; un des premiers mots de la partie affirmative implique une négation : l'adjectif in-nubilus (v. 21), $\alpha \varkappa \alpha \xi \lambda \varepsilon \gamma$ ó $\mu \varepsilon v o v$, création lucrétienne sur le modèle homérique

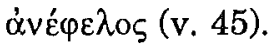

Les vers 21 à 24 finissent d'installer une atmosphère "printanière", de plus en plus opposée à celle de l'hiver, mais aussi (cf. at contra, v. 25) à celle des Enfers, dont le poète prépare la mention (Acherusia templa, v. 25). Lucrèce insiste sur l'absence de nuages et sur la clarté éclatante : un des points communs ${ }^{30}$ entre les tableaux hivernaux et infernaux est justement l'obscurité. Que l'on se souvienne de l'excursus virgilien sur l'hiver en "Scythie», où on lit : Tum sol pallentis haud umquam discutit umbras (G., III, 357). L'expression pallentis... umbras, ainsi que le souligne R. Martin ${ }^{31}$, désigne - plutôt que la nuit polaire ${ }^{32}$ - cet éclairage blafard des ciels obscurcis par les chutes de neige. Cette ambiance inquiétante, où ni soleil ni lumière n'ont place, rappelle celle qui règne au royaume des "ombres» 33 , notamment dans la description qu'en donne Virgile lui-même (Én., VI, 268-316), où l'accent est mis sur le manque de lumière ou la pauvreté de

29 Dans sa conclusion (v. 23-24), Lucrèce reviendra à une proposition négative, qu'il juge plus efficace, sans doute, qu'une simple affirmation.

30 Certaines évocations de l'hiver et des Enfers sont intimement liées, notamment par l'emploi d'un vocabulaire analogue : voir à ce sujet K. PRESTON, Aspects of Autumn in Roman Poetry, in CPh, 13 (1918), p. 272-282, sp. 281-282; I. BoRZSÁK, Stygias detrusus in oras, in AAntHung, 1 (1951-52), p. 459-470; mon étude Letalis hiems, in Grec et latin en 1985 et 1986, textes et documents éd. par Gh. VIRÉ, Bruxelles, 1986, p. 145-153, sp. 146 et 148-149.

31 R. MARTIN, Virgile et la «Scythie» (Géorgiques, III, 349-383), in REL, 44 (1966), p. 286-304, sp. 294.

32 Mentionnée plus loin dans le texte (noctem, v. 379) : voir ma note in Latomus, 46 (1987), p. 211-212.

33 Dans En., IV, 26, Virgile applique l'expression pallentis umbras aux pâles ombres de l'Érèbe. Cf. encore l'ambiance générale du monde souterrain dans En., VI, 534 (sine sole domos); G., IV, 467-484 (caligantem, v. 468; luce carentum, v. 472); Ov., Mét. , IV, 432-436 (nubila, v. 432; nebulas, v. 434); X, 54 (trames, /... caligine densus opaca); MANIL., I, 127 (in infernas caligo pulsa tenebras). Sur les représentations latines des Enfers, voir e.g. Y. FRADIN, Les représentations infernales chez les poètes augustéens, in Caesarodunum, 3 (1969), p. 165-170. 
l'éclairage (v. 268-272) ${ }^{34}$. A vrai dire, le vers 22 (... large diffuso lumine rident) nous renvoie au début du De rerum natura (I, 9 : Placatumque nitet diffuso lumine caelum), soit à l'hymne à Vénus, à l'hymne... au printemps (species... uerna diei, v. 10). Mais Lucrèce ajoute large : le printemps des dieux est un printemps exceptionnel, parce que, à l'inverse du printemps des humains, il n'est jamais supplanté par l'hiver. La conséquence en est une explosion de joie (rident ${ }^{35}$, v. 22), comme dans l'hymne à Vénus (I, 8 : tibi rident aequora pont $i$ ), cette Vénus que fuient vents et nuages (I, $6: T e$, dea, te fugiunt uenti, te nubila caeli; cf. neque... uenti, nec nubila, v. 19 et innubilus, v. 21). Ce rident est la négation absolue de l'hiver, que qualifient de tristis $^{36}$ Virgile (G., IV, 135) et Ovide (Art, I, 407; Ib., 201; $T r .$, III, 10, 9). Enfin, l'abondance de productions (v. 23) est caractéristique du printemps, éventuellement de l'été et de l'automne ${ }^{37}$, mais pas de l'hiver, ce dernier passant aux yeux des poètes pour une saison improductive $^{38}$ ou presque ${ }^{39}$. Comme souvent dans les descriptions du printemps, c'est une négation de l'hiver qui impose la belle saison ${ }^{40}$.

Demandons-nous maintenant pourquoi Lucrèce trace un tableau en aussi nette opposition avec les traits de l'hiver, autrement dit quelles

34 Cf. sp. v. 268 : Ibant obscuri sola sub nocte per umbram.

35 Le texte ici présenté est celui d'A. ERNout (Lucrèce. De la nature. Tome premier, texte ét. et trad., 9e éd. rev. et corr., Paris, 1955), qui adopte rident, correction de Lachmann (cf. p. 115) pour le ridet des manuscrits $(O Q V)-$ sujet : aether -. Dans notre analyse, seuls importent le verbe ridere et sa signification. Sur la question, voir e.g. E.J. KENNEY, op. cit., p. 78-79.

36 Cf. aussi, parmi les épithètes de hiems, dura (VIRG., G. , IV, 239; OV., Tr. , III, 10, 44), fera (Ov., Tr., I, 1, 42) et horrida (Ov., Mét. , XV, 212; Pont. , IV, 10, 38). A propos de tristis, remarquons que Virgile en a fait une épithète de nature du Tartare (Tartara tristia, dans $E n$. IV, 243) et des ombres infernales (tristes umbrae, dans En, V, 734).

37 Cf. LUCR., V, 737-740; VIRG., G., II, 323-324; 521-522; Ov., F., I, 151-156; Rem., 187-188; Priap., 84, 1-2 (B.-H.); CALP. SiCUL., V, 16-25.

38 Cf. VIRG., G., III, 352-353; Ov., Pont. , I, 2, 23; IV, 10, 31; Rem., 188; Tr. , IV, 1, 58; Nux, 127-130; Priap., 84, 2-4 (B.-H.); SÉN., H. O., 1577; LuC., IV, 108; MART, VIII, 68, 10 (sterilis... hiems) et l'ensemble de l'épigramme pour l'idée. En prose, cf. les remarques de Varron ( $L . L, V, 61)$.

39 Certains fruits mûrissent en hiver : les glands, les olives, les arbouses, les baies de laurier et de myrte; cf. LuCR., V, 940-941; VIRG., B., X, 20; G. , I, 305-306; II, 519-520; Priap., 85, 9 (B.-H.); SÉN., Epigr., 3, 4 ( PR. = A L. , 237, 4 [R.]).

40 Cf. e.g. HoR., O., I, 4, 1-4; IV, 12, 1-4; OV., Tr., III, 12, 27-30. 
informations il entend ainsi nous communiquer sur la divinité. Certains éléments ponctuels sont sans doute chargés de symbolisme : l'absence de pluies peut s'interpréter comme une absence de larmes et signifier le parfait bonheur dont jouissent les dieux dans le système épicurien (cf. rident, v. 22) ${ }^{41}$. L'absence de neige blanche, puisque canus s'emploie, je l'ai rappelé, pour qualifier la tête ou les cheveux des vieillards (cf. aussi capitis niues, dans Hor., $O .$, IV, 13, 12), peut exprimer le non-vieillissement des dieux. Mais ceci reste limité et ne nécessitait pas, de la part de Lucrèce, une construction aussi savante ni un rejet de l'hiver en tant que tel.

Nier l'hiver, c'est nier la vieillesse : les Anciens avaient établi une adéquation entre les âges de la vie (enfance, adolescence/jeunesse, maturité, vieillesse) et les saisons de l'année (printemps, été, automne, hiver $)^{42}$. Mais c'est aussi nier la mort; l'hiver était en effet, pour diverses raisons, associé à la mort dans les mentalités des poètes : hiver et mort impliquent tous deux les notions de froid, de destruction et de silence -, mais aussi de fin (l'hiver marque la fin de l'année; la mort, celle de la vie ${ }^{43}$. Or une des propriétés essentielles de la divinité selon les épicuriens est l'immortalitét4. Lucrèce ne fait ici qu'énoncer de façon imagée ce qu'il écrit ailleurs à propos des dieux ${ }^{45}$ :

Omnis enim per se diuom natura necessest

Inmortali aeuo summa cum pace fruatur (II, 646-647).

41 Voir J. B RUN, op. cit., p. 87-88, et P.H. SCHRIJVERs, Horror ac divina voluptas. Études sur la poétique et la poésie de Lucrèce, Amsterdam, 1970, p. 63. Cf. LuCR., II, 646-651; V, 165. Pour tout ce qui concerne la conception et les caractéristiques de la divinité chez les épicuriens, voir A.-J. FESTUGIÈrE, Epicure et ses dieux, 3e éd., Paris, 1985, sp. p. 83-86, 92-94 et 98.

42 Cette idée d'origine pythagoricienne est exposée dans HPC., Hum., 11; ARIST., G. A., V, 3, 784a (17-19); OV., Mét. , XV, 199-213; GALIEN, XVI, p. 26, 345 et 424 (KÜHN); DIOG. LAËRTE, VIII, 10; voir e.g. Fr. BoLL, Die Lebensalter. Ein Beitrag zur antiken Ethologie und zur Geschichte der Zahlen. Mit einem Anhang "Zur

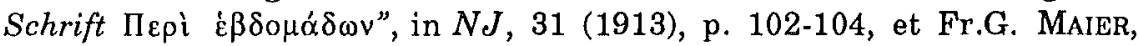
Winteridylle? Erfahrung und Bild des Winters im Altertum, in Neue Zürcher Zeitung, Nr. 299 (Freitag, 23. Dezember 1988), p. 36.

43 Sur l'association hiver-mort, voir mon article Letalis hiems, cité supra, n. 30. Cf. e.g. VIRG., G., IV, 507-527; HoR., O. , IV, 7, 9-16; OV., Mét. , II, 822-828.

44 Voir J. B RUN, op. cit. , p. 88, et P.H. SCHRIJVERS, op. cit. , p. 61.

45 Cf. aussi V, 53 (immortalibu'... diuis) et 165 (inmortalibus). 
Au vers 22, nous lisons le mot lumine, qui, je l'ai souligné, nous remet en mémoire l'hymne à Vénus, hymne au printemps, mais aussi hymne à la vie. Cette lumière, qu'ignorent l'hiver et le royaume des ombres, est celle de la vie ${ }^{46}$ :

Aeneadum genetrix,...

... per te quoniam genus omne animantum

Concipitur, uisitque exortum lumina solis,

...

Efficis ut cupide generatim saecla propagent.

...

Nec sine te quicquam dias in luminis oras

Exoritur (I, 1; 4-5; 20; 22-23).

Souvent lumen ou lux désignent la lumière du jour ou la vie 47 . Inversement, nox peut s'appliquer à la mort (nox perpetua ou aeterna) ${ }^{48}$. Dans notre texte, la «lumière largement répandue» est le symbole de l'éternité divine, mise en valeur par l'exclusion de l'hiver.

En outre, ce climat privilégié n'est autre que celui des temps heureux originels et de l'âge d'or. Les poètes se plaisent à noter qu'alors l'hiver n'existait pas et régnait un éternel printemps ${ }^{49}$. Une conception attestée chez Lucrèce lui-même :

46 Sur l'antinomie de la vie et des ténèbres exprimée par les Grecs et les Latins, voir L. Gueuning, Le thème de la mort dans la poésie d'Horace, in Hum (NRH), 1 (1926), p. 287-310, sp. 302.

47 Cf. e.g. Pl., Cist., 643; TÉr., Héc., 852; LuCR., III, 1033; Cic., Clu., 31; VIrg., En., IV, 31; G. , IV, 255; OV., Tr. , IV, 4, 45; Sil., XIII, 473.

48 Cf. e.g. HoR., O. , I, 4, 16; 28, 15; IV, 9, 27-28; Ov., Hér., X, 112; SÉN., Ad Luc., 82, 16; MART ., X, 71, 5. Catulle, utilisant les deux termes, a produit un heureux effet de contraste (5, 5-6 : Nobis cum semel occidit breuis lux, / Nox est perpetua una dormienda); contraste analogue, mais fondé sur un sens spécifique de lumina, celui d'“yeux" (cf. LUCR., III, 367; V IRG., En., V, 847), dans CATUL., 51, 11-12 (gemina teguntur / lumina nocte) et VIRG., En., X, 746 (=XII, 310 : in aeternam clauduntur lumina noctem).

49 Cf. VIRG., G. II, 338-339 (uer illud erat, uer magnus agebat / orbis); Ov., Mét., I, 107 ( uer erat aeternum); Peruig. Ven., 2 (uere natus orbis est); voir R. GuSTIN, Le printemps chez les poètes latins, in LEC, 15 (1947), p. 323-330, sp. 323-324. Pour d'autres textes où transparaît l'idée d'un printemps originel, voir E. Countney, The Roman Months in Art and Literature, in $M H, 46$ (1988), p. 33-57, sp. 46 : cf. sp. A. L., 874a, 7-8 (R.); PHILoN, Quaest. et solut. in Exodum, I, 1; Spec. Leg., II, 151-152; Pan. Lat., IV, 3, 1; Firm ., Math., III, 1, 17-18; AMBR, Hex. , I, 4, 13; MACR., Comm. , I, 21, 23-24. En ce qui concerne le thème de l'âge d'or stricto sensu, H. REYNEN (Ewiger Frühling und goldene Zeit. Zum Mythos 
At nouitas mundi nec frigora dura ciebat,

Nec nimios aestus, nec magnis uiribus auras (V, 818-819).

Les dieux n'ont pas dépassé le stade - bienheureux - de la jeunesse du monde (nouitas mundi) : nous retrouvons l'idée d'éternelle jeunesse et, tout simplement, celle d'éternité. Il va sans dire que, jouissant d'un climat pareil à celui du "printemps du monde», les dieux connaissent un bonheur parfait.

Dans le tableau lucrétien de la demeure des dieux transparaissent deux grands principes épicuriens en matière de divinité : l'immortalité et la félicité (cf. Épic., Mén., 123 et Diog. Laërte, X, 123 : ròv $\theta \varepsilon$ gò $\zeta \hat{\text { ̣̂ov }}$

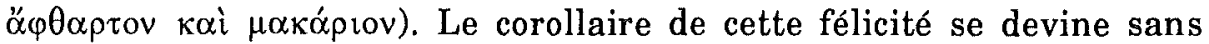
peine : les dieux ne manifesteront aucun intérêt à l'égard du sort des humains ${ }^{50}$. Mais l'insistance sur le climat - tous les traits de la description concernent le climat - permet au poète d'étayer un autre point de son argumentation : les pays présentés comme hivernaux sont d'ordinaire des pays barbares ${ }^{51}$, un des éléments distinguant le barbare du civilisé étant le climat - ceci vaut aussi pour les pays excessivement chauds $^{52}$-. Les Géorgiques en fournissent un bel exemple : au chant II (136-176), Virgile célèbre l'Italie en vantant son climat éternellement printanier (uer adsiduom, v. 149); au chant III, il loue à nouveau sa patrie, implicitement, en utilisant comme repoussoirs la Libye (339-348) et la Scythie (349-383), au climat perpétuellement hivernal (semper hiems, v. 356$)^{53}$. Les dieux de Lucrèce sont des «civilisés» (par opposition aux «barbares»); bénéficiant d'un climat agréable, ce sont des êtres

des goldenen Zeitalters bei Ovid und Vergil, in Gymnasium, 72 [1965], p. 415433, sp. 416) a montré que celui du printemps éternel ne lui était pas associé antérieurement à Ovide, si ce n'est dans un texte isolé de Platon (Pol., 272a).

50 Sur l'indifférence des dieux épicuriens aux hommes et sur leur placidité, voir J. BRUN, op. cit., p. 88; P. BoYANCÉ, op. cit. , p. 219; A.-J. FestugIÈre, op. cit., p. 83-86, 92-94 et 98. Cf. e.g. ÉPIC., Mén., 124; Max. cap., 139 (I); LUCR., II, 648$651 ; \mathrm{V}, 1194-1195 ; \mathrm{VI}, 68-79$.

51 Cf. e.g. Catul., 63, 50-55 et 70; Hor., O., IV, 5, 25; Ov., Pont., IV, 7, 1-12; Tr. , I, 11, 31-44; III, 10, 1-54.

52 Par exemple la Libye, dans VIRG., G., III, 339-348.

53 Voir e.g. E. BuRck, Die Komposition von Vergils Georgika, in Hermes, 64 (1929), p. 279-321, sp. 309-310; Y.A. DAUge, Le Barbare. Recherches sur la conception romaine de la barbarie et de la civilisation, Bruxelles, 1981, p. 152153 et n. 248; mon article La Libye et la Scythie virgiliennes ou l'exotisme au service d'une idéologie, à paraître in Philosophie et exotisme. Colloque Dialogos. Mai 1989 , éd. par B. Decharneux, Bruxelles. 
paisibles (cf. quietae, v. 18) : Lucrèce souligne dans son œuvre qu'ils ne sont pas enclins à la colère ${ }^{54}$. Dès lors, ils ne peuvent plus inspirer la terreur : c'est pour cette raison que Lucrèce écrit diffugiunt animi terrores (v. 16). Le véritable objectif du poète est d'arracher aux hommes leurs vaines craintes (cf. le vers 25, qui balaie la peur des Enfers par une simple négation : nusquam) :

Hunc igitur terrorem animi tenebrasque necessest

Non radii solis neque lucida tela diei

Discutiant, sed naturae species ratioque (I, 146-148).

Cette terreur, contrôlée grâce à la révélation de la doctrine épicurienne, se change en horror (v. 29) ${ }^{55}$, frisson d'extase (cf. diuina uoluptas, v. 28), sorte de peur valorisée, de révérence religieuse pour l'initiateur de cette doctrine ${ }^{56}$, qui est lui-même un deus (cf. deus ille fuit, deus, en V, 8, et hic merito nobis deus esse uidetur, en V, 19).

Lucrèce, j'espère être parvenu à le montrer, construit sa description sur base d'une idée directrice qui se précise graduellement : l'hiver, conçu dans son opposition avec le printemps. Par ce procédé, il pose les grands principes religieux de l'épicurisme : éternité, félicité et indifférence des dieux, que les hommes n'ont pas lieu de redouter. Poésie et philosophie, chez l'auteur du De rerum natura, sont complémentaires.

Route de Philippeville, 33

Pierre-Jacques DEHON

B - 5651 TARCIENNE

54 Cf. II, 651; V, 1194-1195; VI, 68-74.

55 Pour les dénominations de l'angoisse chez Lucrèce, voir J.J.M. ZONNEVELD, Angore metuque. Woordstudie over de angst in De Rerum Natura van Lucretius, Utrecht-Nijmegen, 1959, qui analyse notre passage aux p. 115-117.

56 Cf. SERV., ad Aen., VI, 54-55 (Gelidus Teucris per dura cucurrit / Ossa tremor) : religionis est et diuinae reuerentiae. $\mathrm{R}$. BLOCH (Les prodiges dans l'Antiquité classique [Grèce, Etrurie et Rome], Paris, 1963, p. 71) définit le sentiment d'horror comme «le frisson sacré qui envahit l'âme de l'homme devant le signe tangible et redoutable de l'intervention des forces divines d'où dépend son sorts. 